\title{
Influence of Dietary Phytase, Lysine and Phosphorus on Nutrient Digestibility, Hydrolysis of Phytic Acid and Coenzyme Q10 Synthesis in Laying Hens
}

\author{
IY Martinez Rojas ${ }^{1, *}$, C López Coello², EO Oviedo-Rondón², E Ávila González ${ }^{3}$, J Arce Menocal ${ }^{3}$, and GA Gomes ${ }^{4}$ \\ ${ }^{1}$ Departamento de Medicina y Zootecnia de Aves, Facultad de Medicina Veterinaria y Zootecnia, Universidad Nacional Autónoma de México, México \\ ${ }^{2}$ Prestage Department of Poultry Science, College of Agriculture and Life Sciences, North Carolina State University, Raleigh, USA \\ ${ }^{3}$ Facultad de Medicina Veterinaria y Zootecnia, Universidad Michoacana de San Nicolás Hidalgo, Morelia, México \\ ${ }^{4} \mathrm{AB}$ Vista, Marlborough, Wiltshire, UK
}

*Corresponding author: Martinez Rojas IY, Departamento de Medicina y Zootecnia de Aves, Facultad de Medicina Veterinaria y Zootecnia, Universidad Nacional Autónoma de México, México, Tel: +52-55-2717-9372; Fax: +52-5622-5868; E-mail: ingridmar_1@hotmail.com

Received: 25 Jun, 2020 | Accepted: 10 Jul, 2020 | Published: 13 Jul, 2020

Citation: Martinez Rojas IY, López Coello C, Oviedo-Rondón EO, Ávila González E, Arce Menocal J, et al. (2020) Influence of Dietary Phytase, Lysine and Phosphorus on Nutrient Digestibility, Hydrolysis of Phytic Acid and Coenzyme Q10 Synthesis in Laying Hens. J Anim Sci Res 4(3): dx.doi. org/10.16966/2576-6457.142

Copyright: (C) 2020 Martinez Rojas IY, et al. This is an open-access article distributed under the terms of the Creative Commons Attribution License, which permits unrestricted use, distribution, and reproduction in any medium, provided the original author and source are credited.

\begin{abstract}
High doses of phytase have been shown to increase phytic acid (Phyt-ac) hydrolysis, nutrient availability and coenzyme Q10 (CoQ10) synthesis in broilers, but no previous reports are available in layers. The present study tested the effects of four doses of phytase $(0,300,1200$ and 4800 FTU/ $\mathrm{kg}$ ) and three digestible lysine (dLys) levels $(0.67,0.77$, and $0.87 \%)$ on Phyt-ac hydrolysis, amino acid and mineral digestibility in layers fed diets with $0.12 \%$ available $P$ (avP). Additionally, a diet with $0.25 \%$ avP and $0.87 \%$ dLys, without phytase added, was evaluated as control treatment. CoQ10 content in liver was quantified in the first three doses $(0,300$, and $1200 \mathrm{FTU} / \mathrm{kg})$ at the highest and lowest levels of dLys $(0.67$ and $0.87 \%)$. There was an interaction effect $(\mathrm{P}<0.05)$ between phytase doses and dLys levels for amino acid digestibility. In $0.67 \%$ dLys diets, the response to incremental doses of phytase was linear, while in $0.77 \%$ and $0.87 \%$ dLys there was a quadratic response $(P<0.05)$. P digestibility was higher with incremental doses of phytase, despite a reduced effect of enzyme in $0.87 \%$ dLys diets $(P=0.034)$. No differences $(P>0.05)$ were observed between treatments on availability of $\mathrm{Ca}, \mathrm{Zn}, \mathrm{Cu}, \mathrm{Co}, \mathrm{Mn}$ and Fe. Phytase inclusion increased $\mathrm{Mg}, \mathrm{Na}$, and $\mathrm{K}$ digestibility in 0.67 and $0.77 \%$ dLys diets, but in $0.87 \%$ diets these were reduced $(P<0.05)$. Doses of phytase 1200 and $4800 \mathrm{FTU} / \mathrm{kg}$ increased the presence of lower low inositol esters (Ins-P) such as Ins-5P, Ins-3P and inositol $(P<0.05)$. Ins- $4 \mathrm{P}$ and Ins-2P content was influenced $(P<0.05)$ by phytase addition and dietary dLys. Liver CoQ10 content was similar between phytase doses in $0.67 \%$ dLys diets, but in the $0.87 \%$ dLys diet, phytase addition reduced coenzyme concentration in liver $(P<0.05)$. In conclusion, phytase dose and dLys level in layer diet affected nutrient digestibility, Phyt-ac hydrolyzes, and CoQ10.
\end{abstract}

Keywords: Phytase superdosing; Dietary digestible lysine; lleal digestibility; Hydrolysis of phytic acid; Coenzyme Q10 in liver; Laying hens

\section{Introduction}

The capability of phytic acid (Phyt-ac) to impact the availability of nutrients is due to the presence of 12 replaceable protons with the capacity to associate with other molecules [1]. These chelates in the gastro-intestinal lumen can reduce the absorption and utilization of nutrients such as proteins, amino acids and minerals [2]. Otherwise, the anti-nutritive effect of Phyt-ac can be eliminated by hydrolysis of Phyt-ac covalent bonds by phosphatases, including phytases. During the hydrolysis process are obtained low inositol phosphates (Ins-P), mono-phosphate and free myo-inositol [3]. Previous studies have shown that lower Ins-P esters can negatively affect protein digestibility by limiting solubility and/or blocking digestive enzymes in the gastrointestinal tract [4].

The extent of phytate hydrolysis in the gut will depend on the dietary phytase level. Walk CL, et al. [5] found that using 1500 FTU/ $\mathrm{kg}$, Ins-5P, Ins-4P and Ins-3P content is reduced 33\% compared with $500 \mathrm{FTU} / \mathrm{kg}$, and this last dose is less effective in realizing nutrients. For that, superdosing could highly impact the availability of nutrients, primarily $\mathrm{P}$, but also for protein and mineral [6].

The extra-phosphoric effect of phytase implicates changes in metabolic pathways, modifying metabolic system including coenzymes [7]. Coenzyme Q10 (CoQ10) is recognized as crucial component in the energy producing processes, and an endogenous antioxidant in the cell as well. In the inner mitochondrial membrane, CoQ10 is an electron carrier in the respiratory chain for the formation of ATP. In the complex III of the respiratory chain, CoQ10 is present in three forms: ubiquinone (oxidized-coQ10), ubisemiquinone, and ubiquinol (reduced-CoQ10H ${ }_{2}$ ) [8]. Karadas F, et al. [7] found higher CoQ10 levels in the liver using $12500 \mathrm{FTU} / \mathrm{kg}$ of a 6-phytase derived from Escherichia coli or with $0.45 \%$ avP compare to $0.25 \%$ avP in 21 day old broilers fed with or without $250 \mathrm{FTU} / \mathrm{kg}$ phytase. 
Another variable that can influence the degradation of Phyt-ac is the $\mathrm{P}$ content. Hughes A, et al. [9] reported a negative linear response in phytate ileum digestibility with $0.25 \%$ avP content in diet when testing 200, 400, and $600 \mathrm{FTU} / \mathrm{kg}$ phytase; however, reducing avP to $0.15 \%$, no linear response was observed. In the same study, 500 FTU/ $\mathrm{kg}$ increased phytate digestibility in $0.15 \%$ avP compare to $0.35 \%$ avP. Agbede $\mathrm{JH}$, et al. [10] found higher content of Ins-6P with a dietary content of $0.8 \%$ total $\mathrm{P}$ than $0.4 \%$ in broilers.

Lysine requirements for dietary content have been evaluated in laying hen [11], although there is limited ileal digestibility data available, with no previous evaluation of the impact of phytase superdosing with graded levels of digestible Lys (dLys).

In the present study was hypothesized that phytase superdosing (1200 and $4800 \mathrm{FTU} / \mathrm{kg}$ ) would increase Phyt-ac hydrolysis, improving nutrient digestibility in ileum of laying hens fed a corn-soybean meal diet. Furthermore, was expected that coenzyme Q10 content in liver would be influenced by phytase dose, but not by dietary avP content. Thus, the present study evaluated the effect of inclusion of four doses of a exogenous phytase $(0,300,1200$, and $4800 \mathrm{FTU} / \mathrm{kg})$ in $0.12 \%$ avP diets with three levels of dLys $(0.67,0.77$, and $0.87 \%)$ on ileal digestibility of essential (except tryptophan) and non-essential amino acids, minerals ( $\mathrm{Zn}, \mathrm{Fe}, \mathrm{Mg}, \mathrm{Cu}, \mathrm{Mn}, \mathrm{Ca}, \mathrm{Na}$ and $\mathrm{K}$ ), and on Phytac hydrolysis. Additionally, one diet with $0.25 \%$ avP and $0.87 \%$ dLys was included, with the aim to evaluate the impact of avP level on each one of the above-mentioned variables. The CoQ10 content in liver was quantified for three phytase doses $(0,300$, and $1200 \mathrm{FTU} / \mathrm{kg})$ and two dLys levels ( $0.67 \%$ and $0.87 \%)$.

\section{Materials and Methods}

\section{Experimental design}

Four doses of an exogenous phytase $(0,300,1200$ and 4800 FTU/ $\mathrm{kg}$ ) were tested with three levels of dLys $(0.67,0.77$ and $0.87 \%)$ in a corn-soybean meal based on diet containing $0.12 \%$ avP. The control phytase-free diet with $0.25 \%$ of avP and $0.87 \%$ dLys level was evaluated as well. With the exception of dLys and avP, diets were formulated following the NRC (1994) recommendations for white-line hens (Table 1). Cellulose was used (World Minerals, Lompoc, CA) to obtain all diets with $15 \%$ crude protein, $2.8 \mathrm{M} \mathrm{cal} / \mathrm{kg}$, and $3.5 \%$ of total Ca. The phytase was from Escherichia coli expressed in Trichoderma reesei (Quantum Blue, EC 3.1.3.26, AB Vista, Marlborough, UK) and one FTU was defined as the amount of enzyme required to release $1 \mathrm{~mol}$ of inorganic $\mathrm{P} / \mathrm{min}$ from $0.15 \mathrm{M} / \mathrm{dL}$ of sodium phytate at a temperature of $37^{\circ} \mathrm{C}$ and $5.5 \mathrm{pH}$.

For each treatment, 12 Bovans White laying hens were placed individually in $40 \times 47 \mathrm{~cm}$ cages. Hens had ad libitum access to water, with a maximum feed intake of $105 \mathrm{~g} / \mathrm{hen} /$ day. Facilities were at environmental temperature and hens had a lighting program of 16L: $8 \mathrm{D} \mathrm{h}$. All hen handling procedures were approved by the Institutional Subcommittee for the Care and Use of Experimental Animals (SICUAE) of the Veterinary Medicine Faculty of the National Autonomous University of Mexico.

\section{Digestibility test and sampling}

Layers were fed for 25 weeks with experimental diets and for additional two weeks with the same diets containing $5 \mathrm{~g} / \mathrm{kg}$ titanium dioxide $\left(\mathrm{T}_{\mathrm{i}} \mathrm{O}_{2}\right)$ as an inert marker. For sampling, at age of 65 weeks, all birds were euthanized through intravenous injection of EUTAFIN ${ }^{\circ}$ (390 mg sodium pentobarbital, $50 \mathrm{mg}$ phenytoin sodium and $1 \mathrm{ml}$ excipients) into the radial vein at a dose of $1 \mathrm{~mL} / 5 \mathrm{~kg}$ of BW. The distal ileum sample was collected dissecting the terminal half from the
Meckel's diverticulum portion and remaining up to $2 \mathrm{~cm}$ from before the ileo-cecal valve. Digesta content was flushed out with $5 \mathrm{ml}$ of deionized water, avoiding pressure to the intestinal mucosa. The digesta from three hens were pooled to one sample, for a total of 52 samples ( 4 per diet). Samples were frozen at $-18^{\circ} \mathrm{C}$, and lyophilized for 72 hours.

The gizzard contents were also collected and pooled following the ileal sample order. Contents were homogenized, frozen at $-4^{\circ} \mathrm{C}$, and lyophilized for 72 hours. Finally, the liver was dissected from hens fed with 0,300 and $1200 \mathrm{FTU} / \mathrm{kg}$ phytase in the diets with 0.67 and $0.87 \%$ $\mathrm{dLys}$ and from the control diet; they were frozen at $-40^{\circ} \mathrm{C}$.

\section{Laboratory analysis}

Feed: Protein, total lysine, $\mathrm{P}$ and Ca contents were analyzed in the basal diet following AOAC (2006) techniques. Exogenous phytase activity in feed samples was analyzed by Enzyme Services and Consultancy (ESC, YstradMynach, UK). Essential and non-essential amino acids, except for tryptophan, were quantified in feed and ileal digesta. Briefly, samples were oxidized using a solution of hydrogen peroxide, formic acid and phenol, and were hydrolyzed with $6 \mathrm{M}$ hydrochloric acid for 24 hours at $110^{\circ} \mathrm{C}$. Ion exchange chromatography technique was used to separate amino acids, with post-column quantification at $570 \mathrm{~nm}$ of ninhydrin ( $440 \mathrm{~nm}$ for proline).

For mineral analysis, samples were incinerated at $500^{\circ} \mathrm{C}$ for $8 \mathrm{~h}$, and $\mathrm{P}, \mathrm{Ca}, \mathrm{Zn}, \mathrm{Cu}, \mathrm{Co}, \mathrm{Mn}, \mathrm{Mg}, \mathrm{Fe}, \mathrm{K}$, and $\mathrm{Na}$, and quantified by atomic absorption spectrophotometry technique, following the AOAC (2006b) procedures. The apparent ileal digestibility coefficient (AIDC) of each amino acids and mineral was estimated using the next formulation:

$\mathrm{AIDC}=\left[\left(\right.\right.$ nutrient $\left./ \mathrm{TiO}_{2}\right) \quad \operatorname{diet}-\left(\right.$ nutrient $\left./ \mathrm{TiO}_{2}\right)$ ileum $] /($ nutrient$/$
$\left.\mathrm{TiO}_{2}\right)$ diet

Low inositol esters: Inositol phosphate esters and inositol were determined by high-performance ion chromatography [12].

Coenzyme Q10: The coenzyme content was determined in pools of three livers, gathered for ileal digesta, giving a total of 28 samples. The extraction of CoQ10 was done following the methodology described for Karadas F, et al. [7], and CoQ10 was quantified as Mattila P and Kumpulainen J [13]. Briefly, the pooled livers were liquefied and mixed with $5 \%\left(\mathrm{w} / \mathrm{v}\right.$ in $\left.\mathrm{H}_{2} \mathrm{O}\right)$ solution of $\mathrm{NaCl}(0.7 \mathrm{ml})$ and ethanol $(1 \mathrm{ml})$ to separate coenzyme in the hexane phase. The extraction was done twice and the hexane phase was combined, evaporated and red is solved in a mixture of methanol/dichloromethane $(1: 1, \mathrm{v} / \mathrm{v})$. Coenzyme Q10 was analyzed by HPLC using a Hewlett-Packard 1100 equipped with a Vydac 201TP54 column ( $5 \mu \mathrm{m}, 250 \times 4.6 \mathrm{~mm}$, the Separations Goup, U.S.A). The mobile phase was methanol/2-propanol/ethanol (70:15:15, $\mathrm{v} / \mathrm{v} / \mathrm{v}$ ), with a flow rate of $0.8 \mathrm{ml} / \mathrm{min}$ and the injection volume was 50 $\mu \mathrm{L}$. Instrumentation was controlled by the HP 3D Chem Station computer program Rev. A. 06.01. The standard employed was Coenzyme Q10 \#C9538-1G from Sigma Chemicals Co., U.S.A.

Statistical analysis: Treatments with $0.12 \%$ avP were analyzed as factorial arrangement of treatments nested in dietary avP content through Fit Model option of JMP program, categorizing the factors as nominal variables. Further more, avP level was added as source of variation in the ANOVA. Means were separated using the methodology of Tukey or MDS adjusted taking $\mathrm{p}$ value as 0.07 .The statistical model was:

\section{$\mathrm{Y}=\mu+\gamma \mathrm{i}+[\alpha \mathrm{m}+\beta \mathrm{n}+\alpha \beta \mathrm{mn}] \mathrm{j}(\mathrm{i})+\varepsilon(\mathrm{ij}) \mathrm{k}$}

Where $\mu$ is the overall mean, $\gamma$ is $i^{\text {th }}$ level of avP level, $\alpha$ is the effect of $\mathrm{m}^{\text {th }}$ level of dLys, $\beta$ is the effect of $\mathrm{n}^{\text {th }}$ dose of phytase and $\varepsilon$ is the 
experimental error. Regression analysis was applied to continuous values of the factors phytase dose and dLys content, using Fit Model at JMP ${ }^{\circledR}$ program.

\section{Results}

There was an interaction between dLys level and phytase dose in digestibility of essential and non-essential amino acids ( $<<0.05$; Table 2 and 3). In the $0.67 \%$ dLys level, increasing levels of phytase resulted in a linear positive response in most amino acids, except for Thr. In the $0.77 \%$ dLys level, linear positive response was observed for Arg, His, Ile, Lys, and Val, and a quadratic response for Leu and Met, and

Table 1: Composition of basal experimental diet and analyzed nutrient content.

\begin{tabular}{|c|c|}
\hline Ingredient & Kilograms \\
\hline Yellow corn (8\%) & 690.01 \\
\hline Soybean meal (48\%) & 199.51 \\
\hline Calcium carbonate & 94.31 \\
\hline Salt (NaCl) & 4.01 \\
\hline Cellulose & 3 \\
\hline Vitamins and minerals ${ }^{1}$ & 2.41 \\
\hline DL- methionine $84 \%$ & 1.61 \\
\hline Ortophosphate 18:21 & 1.31 \\
\hline Yellow pigment $15 \mathrm{~g} / \mathrm{Kg}$ (Tagetes erecta) & 1.01 \\
\hline Red pigment $5 \mathrm{~g} / \mathrm{Kg}$ (Capsicum annuum) & 0.81 \\
\hline Choline chloride $60 \%$ & 0.81 \\
\hline BMD-100² & 0.51 \\
\hline Cyromazine 1\% & 0.51 \\
\hline Antioxidant $^{2}$ & 0.21 \\
\hline L- Lysine $76.4 \%$ & 0.01 \\
\hline Escherichia coli phytase & 0 \\
\hline \multicolumn{2}{|l|}{ Analyzed nutrient content } \\
\hline Crude protein $(\%)^{+}$ & 15.1 \\
\hline Metabolizable energy $(\mathrm{Mcal} / \mathrm{kg})^{+}$ & 2.8 \\
\hline Digestible methionine and cysteine $(\%)^{\dagger+}$ & 0.65 \\
\hline Digestible methionine $(\%)^{\dagger+}$ & 0.38 \\
\hline Digestible lysine $(\%)^{+}$ & 0.67 \\
\hline Digestible threonine $(\%)^{+\dagger}$ & 0.61 \\
\hline Total calcium $(\%)^{+}$ & 3.5 \\
\hline Total phosphorus $(\%)^{+}$ & 0.34 \\
\hline Available phosphorus $(\%)^{+}$ & 0.12 \\
\hline
\end{tabular}

${ }^{\dagger}$ Value analyzed; ${ }^{+\dagger}$ Value calculated

${ }^{1}$ Vitamin premix provided Vitamin A 10,000,000 IU; Vitamin D3 2,500,000 IU; Vitamin E 6,000 IU; Vitamin K 2.5 g; Thymine 1.6 g; Riboflavin 5 g; Cyanocobalamin $0.10 \mathrm{~g}$, Folic acid $0.50 \mathrm{~g}$; Pyridoxine $1.5 \mathrm{~g}$; Calcium pantothenate $10 \mathrm{~g}$; Niacina $30 \mathrm{~g}$; Choline chloride $60 \% 200 \mathrm{~g}$, Iron $80 \mathrm{~g}$; Manganese 60g; Copper $10 \mathrm{~g}$; lodine $0.3 \mathrm{~g}$; Zinc $50 \mathrm{~g}$; Selenium $0.30 \mathrm{~g}$; Antioxidant $125 \mathrm{~g}$; Vehicle c.b.p 1,000,000 g per kg diet.

${ }^{2} \mathrm{BHA}$ (Butyl hydroxy anisole) 1.2\%; BHT (Butyl hidroxy toluene) 9.0\%; Ethoxiquine 4.8\%; Chelating agents 10 no trend was tracked for Cys and Phe. There was less significance with $0.87 \%$ dLys, where Arg, Cys, Ile, and Val exhibited a quadratic tendency, while the digestibility of other essential amino acid was not influenced. In the case of Thr, digestibility was best described by mainly effect of both factors, a linear positive response to dLys level and by quadratic response to phytase dose (Table 2).

About non-essential amino acid digestibility, the response to phytase dose was linear at $0.67 \%$ dLys level for Ala, Asp, Glu, Gly, Ser, and Pro. At $0.77 \%$ dLys, Pro was the only non-essential amino acid that did not show a linear response. Furthermore, there was a quadratic response to phytase dose in $0.87 \%$ dLys, except for Ala and Pro ( $\mathrm{p}<0.05$; Table 3 ). In ANOVA analyses, level of $0.25 \%$ of avP in the diet decreased Asp and His digestibility, while Tyr digestibility was increased ( $\mathrm{p}<0.05$; Table 2 and 3 ).

Digestibility of $\mathrm{P}$ was improved with incremental doses of phytase in 0.67 and $0.77 \%$ dLys diets ( $\mathrm{p}=0.06$ ). In $0.87 \%$ dLys diets, $4800 \mathrm{FTU} /$ $\mathrm{kg}$ increased $\mathrm{P}$ digestibility regards to no phytase inclusion at both avP content and regards to $300 \mathrm{FTU} / \mathrm{kg}$ phytase dose. Furthermore, $1200 \mathrm{FTU} / \mathrm{kg}$ showed higher P digestibility than phytase-free $0.12 \%$ avP diet.

$\mathrm{Mg}$ digestibility increased with phytase addition, particularly in the $0.67 \%$ dLys diet, but positive significance was less evident in $0.77 \%$ dLys level. Otherwise, it decreased in the $0.87 \%$ dLys diet $(\mathrm{p}<0.05)$. Increasing dLys content to $0.87 \%$ reduced the positive effect of phytase inclusion on $\mathrm{K}$ and $\mathrm{Na}$ digestibility $(\mathrm{p}<0.05)$. Digestibility of $\mathrm{Na}$ fit to linear and quadratic responses in diets with $0.67 \%$ and $0.77 \%$ dLys, respectively. Inclusion of inorganic $\mathrm{P}$ of $0.25 \%$ avP gave further improvements in digestibility of $\mathrm{Ca}, \mathrm{Zn}, \mathrm{Cu}, \mathrm{Fe}, \mathrm{Mg}, \mathrm{K}$ and Na relative to the group of $0.12 \%$ avP diets ( $\mathrm{p}<0.05$; Table 4$)$.

Phytase inclusion significantly reduced Ins-6P gizzard concentrations, with statistical differences between the doses 4800 FTU/kg and $300 \mathrm{FTU} / \mathrm{kg}$ ( $\mathrm{p}<0.001) .0 .25 \%$ avP diet had higher Ins-6P amount relative to $0.67 \%$ and $0.77 \%$ dLys in $0.12 \%$ avP diets $(\mathrm{p}<0.05)$. Gizzard concentration of Ins-5P in the $0.25 \%$ avP diet was higher than the obtained in $0.12 \%$ avP phytate-free diet with $0.87 \%$ dLys. Doses of 1200 and $4800 \mathrm{FTU} / \mathrm{kg}$ phytase gave lower gizzard concentrations of Ins-5P than phytase-free diets with $0.12 \%$ avP, while $300 \mathrm{FTU} / \mathrm{kg}$ reduced the concentration only to $0.25 \%$ avP diet without phytase $(\mathrm{p}<0.001)$. Furthermore, gizzard Ins-5P levels in diets with $0.12 \%$ avP had less content with $0.67 \%$ and $0.77 \%$ dLys diets than $0.87 \%$ dLys; in addition, this last diet had lower concentrations of Ins-5P than the $0.25 \%$ avP diet $(\mathrm{p}<0.05$; table 5$)$.

A significant interaction between phytase doses and dLys levels was observed for gizzard Ins-4P content; the phytase-free diet in $0.87 \%$ dLys level gave a higher gizzard content of Ins-4P $(p<0.001)$. Increasing levels of phytase resulted in reductions of the gizzard Ins- $4 \mathrm{P}$ concentration with decreasing dietary dLys levels $(\mathrm{p}<0.001$; Table 5).

Content of Ins-3P was higher in the phytate-free $0.25 \%$ avP diet than in $0.12 \%$ avP diets with 1200 and 4800 FTU phytase added. Phytase inclusion at 1200 and $4800 \mathrm{FTU} / \mathrm{kg}$ resulted in lower gizzard Ins-3P concentrations compared to $300 \mathrm{FTU} / \mathrm{kg}$ in $0.12 \%$ avP diets $(\mathrm{p}<0.001)$. Diets with $0.77 \%$ dLys had lower gizzard contents of Ins-3P than $0.67 \%$ and $0.87 \%$ dLys diets with $0.12 \%$ avP, but not for $0.87 \%$ dLys diets with $0.25 \%$ avP $(\mathrm{p}=0.001)$. For gizzard contents of Ins$2 \mathrm{P}$ there was a phytase: dLys interaction, where in $0.67 \%$ dLys diets increases of phytase decreased the content of Ins-2P, while in $0.77 \%$ and $0.87 \%$ diets Ins-2P content increased. Diet with $0.25 \%$ avP level gave higher gizzard content of Ins-2P than the average of the diets with 
Table 2: Effect of addition of four doses of phytase in three graded levels of digestible lysine on apparent ileal digestibility coefficients of essential amino acids in 67 wk-old White Bovans laying hens.

\begin{tabular}{|c|c|c|c|c|c|c|c|c|c|c|c|c|}
\hline AvP (\%) & $\begin{array}{c}\text { Phytase } \\
\text { (FTU/ } \\
\text { kg) }\end{array}$ & $\begin{array}{l}\text { Dig. } \\
\text { Lysine } \\
(\%)\end{array}$ & Arg & Cys & His & Ile & Leu & Lys & Met & Phe & Thr & Val \\
\hline \multirow{12}{*}{0.12} & 0 & \multirow{4}{*}{0.67} & $0.88^{d}$ & $0.69^{a b}$ & $0.86^{\mathrm{ab}}$ & $0.83^{a b c}$ & $0.86^{a b}$ & $0.84^{b}$ & $0.90^{\mathrm{ab}}$ & $0.86^{\mathrm{abc}}$ & $0.75^{\mathrm{ab}}$ & $0.81^{\mathrm{ab}}$ \\
\hline & 300 & & $0.84^{\mathrm{e}}$ & $0.64^{b}$ & $0.80^{c}$ & $0.78^{c}$ & $0.83^{b}$ & $0.77^{c}$ & $0.88^{b}$ & $0.82^{c}$ & $0.67^{b}$ & $0.76^{\mathrm{b}}$ \\
\hline & 1200 & & $0.90^{\mathrm{abcd}}$ & $0.73^{\mathrm{ab}}$ & $0.86^{a b}$ & $0.86^{\mathrm{ab}}$ & $0.88^{a}$ & $0.86^{\mathrm{ab}}$ & $0.91^{\mathrm{ab}}$ & $0.87^{\mathrm{abc}}$ & $0.77^{a}$ & $0.83^{\mathrm{ab}}$ \\
\hline & 4800 & & $0.92^{\mathrm{a}}$ & $0.79^{a}$ & $0.90^{\mathrm{a}}$ & $0.89^{a}$ & $0.91^{\mathrm{a}}$ & $0.90^{\mathrm{a}}$ & $0.94^{a}$ & $0.91^{\mathrm{a}}$ & $0.82^{\mathrm{a}}$ & $0.87^{a}$ \\
\hline & 0 & \multirow{4}{*}{0.77} & $0.90^{\mathrm{abcd}}$ & $0.73^{\mathrm{ab}}$ & $0.87^{a b}$ & $0.85^{\mathrm{ab}}$ & $0.88^{a b}$ & $0.89^{a b}$ & $0.91^{\mathrm{ab}}$ & $0.88^{\mathrm{abc}}$ & $0.77^{a}$ & $0.83^{\mathrm{ab}}$ \\
\hline & 300 & & $0.91^{\mathrm{abc}}$ & $0.77^{a}$ & $0.87^{a b}$ & $0.86^{\mathrm{ab}}$ & $0.88^{\mathrm{ab}}$ & $0.87^{a b}$ & $0.92^{\mathrm{ab}}$ & $0.88^{\mathrm{abc}}$ & $0.79^{a}$ & $0.84^{a}$ \\
\hline & 1200 & & $0.92^{\mathrm{abc}}$ & $0.78^{a}$ & $0.88^{\mathrm{ab}}$ & $0.88^{\mathrm{ab}}$ & $0.90^{\mathrm{a}}$ & $0.89^{\mathrm{ab}}$ & $0.93^{a}$ & $0.90^{\mathrm{ab}}$ & $0.80^{\mathrm{a}}$ & $0.86^{a}$ \\
\hline & 4800 & & $0.92^{\mathrm{a}}$ & $0.76^{a}$ & $0.89^{\mathrm{ab}}$ & $0.88^{\mathrm{ab}}$ & $0.90^{\mathrm{a}}$ & $0.91^{\mathrm{a}}$ & $0.93^{a}$ & $0.88^{\mathrm{abc}}$ & $0.80^{a}$ & $0.88^{\mathrm{ab}}$ \\
\hline & 0 & \multirow{5}{*}{0.87} & $0.89^{c d}$ & $0.71^{\mathrm{ab}}$ & $0.87^{\mathrm{ab}}$ & $0.83^{\mathrm{abc}}$ & $0.86^{\mathrm{ab}}$ & $0.88^{\mathrm{ab}}$ & $0.91^{\mathrm{ab}}$ & $0.83^{b c}$ & $0.74^{\mathrm{ab}}$ & $0.81^{\mathrm{ab}}$ \\
\hline & 300 & & $0.92^{\mathrm{ab}}$ & $0.76^{a}$ & $0.89^{a b}$ & $0.87^{a b}$ & $0.89^{a}$ & $0.90^{\mathrm{ab}}$ & $0.92^{\mathrm{ab}}$ & $0.87^{\mathrm{abc}}$ & $0.79^{a}$ & $0.85^{a}$ \\
\hline & 1200 & & $0.91^{\mathrm{abc}}$ & $0.77^{a}$ & $0.87^{a b}$ & $0.86^{\mathrm{ab}}$ & $0.89^{a}$ & $0.90^{\mathrm{ab}}$ & $0.92^{\mathrm{ab}}$ & $0.87^{\mathrm{abc}}$ & $0.78^{a}$ & $0.85^{a}$ \\
\hline & 4800 & & $0.90^{\mathrm{bcd}}$ & $0.72^{\mathrm{ab}}$ & $0.84^{b c}$ & $0.83^{b c}$ & $0.86^{a b}$ & $0.87^{\mathrm{ab}}$ & $0.90^{\mathrm{ab}}$ & $0.86^{a b c}$ & $0.75^{a}$ & $0.81^{\mathrm{ab}}$ \\
\hline \multirow[t]{2}{*}{0.25} & 0 & & $0.89^{\mathrm{cd}}$ & $0.75^{a}$ & $0.85^{b c}$ & $0.83^{\mathrm{ab}}$ & $0.87^{a b}$ & $0.87^{\mathrm{ab}}$ & $0.91^{\mathrm{ab}}$ & $0.86^{\mathrm{abc}}$ & $0.75^{\mathrm{ab}}$ & $0.82^{\mathrm{ab}}$ \\
\hline & & SEM & 0.005 & 0.012 & 0.006 & 0.007 & 0.006 & 0.007 & 0.006 & 0.008 & 0.010 & 0.009 \\
\hline \multicolumn{13}{|c|}{ Main Effect Means } \\
\hline \multirow{4}{*}{0.12} & 0 & & $0.89^{b c}$ & $0.71^{\mathrm{b}}$ & $0.87^{a b}$ & $0.84^{\mathrm{ab}}$ & $0.87^{b}$ & $0.87^{a b}$ & 0.91 & $0.86^{\mathrm{b}}$ & $0.76^{\mathrm{ab}}$ & $0.82^{b}$ \\
\hline & 300 & & $0.89^{c}$ & $0.72^{\mathrm{ab}}$ & $0.85^{b}$ & $0.84^{b}$ & $0.86^{b}$ & $0.86^{b}$ & 0.91 & $0.86^{\mathrm{b}}$ & $0.75^{b}$ & $0.82^{b}$ \\
\hline & 1200 & & $0.91^{\mathrm{ab}}$ & $0.76^{\mathrm{a}}$ & $0.87^{a}$ & $0.86^{\mathrm{a}}$ & $0.89^{a}$ & $0.88^{a}$ & 0.92 & $0.88^{a}$ & $0.78^{\mathrm{ab}}$ & $0.85^{\mathrm{ab}}$ \\
\hline & 4800 & & $0.91^{\mathrm{a}}$ & $0.76^{\mathrm{ab}}$ & $0.87^{a}$ & $0.87^{a}$ & $0.89^{a}$ & $0.89^{\mathrm{a}}$ & 0.92 & $0.88^{a}$ & $0.79^{a}$ & $0.85^{\mathrm{a}}$ \\
\hline 0.25 & 0 & & $0.90^{\mathrm{abc}}$ & $0.75^{\mathrm{ab}}$ & $0.85^{b}$ & $0.83^{\mathrm{ab}}$ & $0.87^{a b}$ & $0.87^{a}$ & 0.91 & $0.86^{\mathrm{ab}}$ & $0.75^{\mathrm{ab}}$ & $0.82^{\mathrm{ab}}$ \\
\hline \multirow{3}{*}{0.12} & & 0.67 & $0.89^{b} \mathbf{L}$ & $0.71^{b} \mathbf{L}$ & $0.85^{b} \mathbf{L}$ & $0.84^{b} \mathrm{~L}$ & $0.87^{b} \mathbf{L}$ & $0.84^{\mathrm{b}} \mathbf{L}$ & $0.90^{\mathrm{b}} \mathbf{L}$ & $0.87^{\mathrm{ab}} \mathbf{L}$ & $0.75^{b}$ & $0.82^{b} \mathbf{L}$ \\
\hline & & 0.77 & $0.91^{a} \mathbf{L}$ & $0.76^{a}$ & $0.88^{\mathrm{a}} \mathrm{L}$ & $0.87^{\mathrm{a}} \mathbf{L}$ & $0.89^{\mathrm{a}} \mathbf{Q}$ & $0.89^{\mathrm{a}} \mathbf{L}$ & $0.92^{\mathrm{a}} \mathbf{Q}$ & $0.88^{a}$ & $0.79^{a}$ & $0.85^{\mathrm{a}} \mathbf{L}$ \\
\hline & & \multirow{2}{*}{0.87} & $0.92^{\mathrm{a}} \mathbf{Q}$ & $0.74^{\mathrm{ab}} \mathbf{Q}$ & $0.87^{\mathrm{ab}}$ & $0.85^{\mathrm{ab}} \mathbf{Q}$ & $0.87^{a b}$ & $0.89^{\mathrm{a}}$ & $0.91^{\mathrm{ab}}$ & $0.86^{b}$ & $0.77^{\mathrm{ab}}$ & $0.83^{b} \mathbf{Q}$ \\
\hline 0.25 & & & $0.89^{\mathrm{ab}}$ & $0.75^{\mathrm{ab}}$ & $0.85^{b}$ & $0.83^{\mathrm{ab}}$ & $0.87^{a b}$ & $0.87^{a b}$ & $0.91^{\mathrm{ab}}$ & $0.86^{\mathrm{ab}}$ & $0.75^{\mathrm{ab}}$ & $0.82^{\mathrm{ab}}$ \\
\hline 0.12 & & & 0.90 & 0.74 & $0.87^{b}$ & 0.85 & 0.88 & 0.87 & 0.91 & 0.87 & 0.77 & $0.83^{b}$ \\
\hline \multirow[t]{6}{*}{0.25} & & & 0.89 & 0.75 & $0.85^{a}$ & 0.83 & 0.87 & 0.87 & 0.91 & 0.86 & 0.75 & $0.82^{\mathrm{a}}$ \\
\hline & \multicolumn{12}{|c|}{$p$-values } \\
\hline & & & 0.001 & $0.011^{*}$ & $0.048 *$ & $0.004 * *$ & $0.002 * *$ & $<.001$ & 0.088 & 0.019* & $0.009 * * \mathbf{Q}$ & $0.004 * *$ \\
\hline & & & 0.001 & $0.008 * *$ & $0.008^{* *}$ & $0.010 *$ & $0.017^{*}$ & $<.001$ & $0.037^{*}$ & $0.026 *$ & $0.007^{* *} \mathbf{L}$ & $0.009 * *$ \\
\hline & Lysin & tase & $<0.001$ & $0.006 * *$ & $<0.001$ & $<0.001$ & 0.001 & $<.001$ & $0.008 *$ & $0.018^{*}$ & $<0.001$ & 0.001 \\
\hline & & & 0.353 & 0.620 & $0.063^{*}$ & 0.112 & 0.513 & 0.974 & 0.936 & 0.666 & 0.323 & 0.582 \\
\hline
\end{tabular}

$0.12 \%$ avP $(\mathrm{p}<0.001$; Table 5$)$. Layers fed with $1200 \mathrm{FTU} / \mathrm{kg}$ had higher gizzard digest a levels of inositol than those fed with phytase-free diets with $0.12 \%$ and $0.25 \%$ avP levels. Additionally, $0.25 \%$ avP diet had lower gizzard inositol content than $0.12 \%$ avP diets $(\mathrm{p}<0.05$; Table 5).

In $0.67 \%$ dLys, there were no differences between phytase doses in CoQ10 content, but in $0.87 \%$ phytase-free diet resulted in higher CoQ10 liver content than 300 and $1200 \mathrm{FTU} / \mathrm{kg}$ doses. Including $1200 \mathrm{FTU} / \mathrm{kg}$ in $0.87 \%$ dLys level reduced the CoQ10 concentration compare to 0,300 and $1200 \mathrm{FTU} / \mathrm{kg}$ phytase addition $(\mathrm{p}<0.001)$. Furthermore, $0.25 \%$ avP diet gave lower liver content than the average of the $0.12 \%$ avP diets ( $\mathrm{p}=0.001$; Table 5$)$.

\section{Discussion and Conclusion}

In layers previous studies regard to amino acid digestibility effect of phytase are few and results are generally inconclusive. While Jalal MA, et al. [14] observed effect on the digestibility of Met, Cys, Ala, 
Table 3: Effect of addition of four doses of phytase in three graded levels of digestible lysine on apparent ileal digestibility of non-essential amino acids in 67 wk-old White Bovans laying hens.

\begin{tabular}{|c|c|c|c|c|c|c|c|c|c|}
\hline AvP (\%) & Phytase (FTU/kg) & Diges. Lysine (\%) & Ala & Asp & Glu & Gly & Pro & Ser & Tyr \\
\hline \multirow{12}{*}{0.12} & 0 & \multirow{4}{*}{0.67} & C $0.83^{\mathrm{abcd}}$ & $0.82^{\mathrm{abc}}$ & $0.87^{\mathrm{ab}}$ & $0.78^{a b}$ & $0.83^{\mathrm{ab}}$ & $0.81^{\mathrm{ab}}$ & $0.85^{\mathrm{ab}}$ \\
\hline & 300 & & $0.79^{d}$ & $0.77^{c}$ & $0.85^{b}$ & $0.71^{\mathrm{b}}$ & $0.80^{\mathrm{b}}$ & $0.75^{b}$ & $0.82^{\mathrm{ab}}$ \\
\hline & 1200 & & C $0.85^{\mathrm{abcd}}$ & $0.84^{\mathrm{ab}}$ & $0.89^{a b}$ & $0.79^{a b}$ & $0.85^{a b}$ & $0.83^{a}$ & $0.84^{\mathrm{ab}}$ \\
\hline & 4800 & & $0.90^{\mathrm{a}}$ & $0.88^{a}$ & $0.92^{\mathrm{a}}$ & $0.84^{\mathrm{ab}}$ & $0.89^{a}$ & $0.86^{a}$ & $0.89^{a}$ \\
\hline & 0 & \multirow{4}{*}{0.77} & $0.84^{\mathrm{abcd}}$ & $0.84^{\mathrm{ab}}$ & $0.88^{\mathrm{ab}}$ & $0.80^{a}$ & $0.85^{\mathrm{ab}}$ & $0.83^{a}$ & $0.86^{\mathrm{ab}}$ \\
\hline & 300 & & $0.84^{\mathrm{abcd}}$ & $0.85^{\mathrm{ab}}$ & $0.90^{\mathrm{ab}}$ & $0.80^{\mathrm{a}}$ & $0.87^{a}$ & $0.84^{a}$ & $0.87^{a}$ \\
\hline & 1200 & & $0.87^{a b c}$ & $0.86^{\mathrm{ab}}$ & $0.91^{\mathrm{a}}$ & $0.83^{a}$ & $0.88^{a}$ & $0.86^{a}$ & $0.87^{a}$ \\
\hline & 4800 & & $0.88^{\mathrm{ab}}$ & $0.87^{\mathrm{ab}}$ & $0.91^{\mathrm{a}}$ & $0.84^{a}$ & $0.88^{a}$ & $0.86^{a}$ & $0.84^{\mathrm{ab}}$ \\
\hline & 0 & \multirow{5}{*}{0.87} & $0.81^{\text {cd }}$ & $0.82^{\mathrm{abc}}$ & $0.87^{a b}$ & $0.77^{\mathrm{ab}}$ & $0.83^{\mathrm{ab}}$ & $0.81^{\mathrm{ab}}$ & $0.80^{b}$ \\
\hline & 300 & & $0.86^{\mathrm{abcd}}$ & $0.86^{\mathrm{ab}}$ & $0.90^{\mathrm{a}}$ & $0.82^{\mathrm{a}}$ & $0.86^{\mathrm{ab}}$ & $0.84^{a}$ & $0.83^{\mathrm{ab}}$ \\
\hline & 1200 & & $0.86^{\mathrm{abcd}}$ & $0.85^{\mathrm{ab}}$ & $0.90^{\mathrm{a}}$ & $0.81^{\mathrm{a}}$ & $0.87^{a}$ & $0.85^{a}$ & $0.87^{\mathrm{ab}}$ \\
\hline & 4800 & & $0.82^{\mathrm{bcd}}$ & $0.82^{\mathrm{abc}}$ & $0.88^{\mathrm{ab}}$ & $0.77^{\mathrm{a}}$ & $0.85^{\mathrm{ab}}$ & $0.82^{\mathrm{a}}$ & $0.86^{\mathrm{ab}}$ \\
\hline \multirow[t]{2}{*}{0.25} & 0 & & $0.85^{\text {abcd }}$ & $0.81^{b c}$ & $0.87^{a b}$ & $0.78^{\mathrm{ab}}$ & $0.85^{\mathrm{ab}}$ & $0.82^{\mathrm{a}}$ & $0.89^{a}$ \\
\hline & \multicolumn{2}{|c|}{ Sem } & 0.008 & 0.007 & 0.006 & 0.010 & 0.008 & 0.008 & 0.008 \\
\hline \multicolumn{10}{|c|}{ Main Effect Means } \\
\hline \multirow{4}{*}{0.12} & 0 & & $0.83^{c}$ & $0.83^{\mathrm{ab}}$ & $0.88^{b}$ & $0.78^{\mathrm{ab}}$ & $0.84^{\mathrm{b}}$ & $0.81^{b c}$ & $0.84^{b}$ \\
\hline & 300 & & $0.83^{b c}$ & $0.83^{\mathrm{ab}}$ & $0.90^{\mathrm{ab}}$ & $0.78^{b}$ & $0.84^{\mathrm{ab}}$ & $0.81^{c}$ & $0.84^{b}$ \\
\hline & 1200 & & $0.86^{\mathrm{ab}}$ & $0.85^{a}$ & $0.89^{a b}$ & $0.81^{\mathrm{ab}}$ & $0.87^{a b}$ & $0.84^{\mathrm{ab}}$ & $0.86^{\mathrm{ab}}$ \\
\hline & 4800 & & $0.87^{a}$ & $0.86^{a}$ & $0.87^{a}$ & $0.82^{a}$ & $0.87^{a}$ & $0.85^{a}$ & $0.86^{\mathrm{ab}}$ \\
\hline 0.25 & 0 & & $0.85^{\mathrm{abc}}$ & $0.81^{\mathrm{b}}$ & $0.87^{a}$ & $0.78^{\mathrm{ab}}$ & $0.85^{\mathrm{ab}}$ & $0.82^{\mathrm{abc}}$ & $0.89^{a}$ \\
\hline \multirow{3}{*}{0.12} & & 0.67 & $0.84^{\mathrm{ab}} \mathbf{L}$ & $0.83^{b} \mathrm{~L}$ & $0.88^{b} \mathrm{~L}$ & $0.78^{b} \mathrm{~L}$ & $0.84^{\mathrm{ab}} \mathbf{L}$ & $0.81^{\mathrm{b}} \mathrm{L}$ & $0.85^{b}$ \\
\hline & & 0.77 & $0.86^{\mathrm{a}} \mathbf{L}$ & $0.86^{\mathrm{a}} \mathbf{L}$ & $0.90^{\mathrm{a}} \mathbf{L}$ & $0.82^{\mathrm{a}} \mathbf{L}$ & $0.87^{\mathrm{ab}} \mathbf{Q}$ & $0.84^{\mathrm{a}} \mathbf{L}$ & $0.86^{\mathrm{ab}}$ \\
\hline & & \multirow{2}{*}{0.87} & $0.84^{b}$ & $0.84^{\mathrm{ab}} \mathbf{Q}$ & $0.90^{\mathrm{ab}} \mathbf{Q}$ & $0.79^{\mathrm{ab}} \mathbf{Q}$ & $0.85^{a}$ & $0.83^{\mathrm{ab}} \mathbf{Q}$ & $0.84^{\mathrm{ab}}$ \\
\hline 0.25 & & & $0.85^{\mathrm{ab}}$ & $0.81^{\mathrm{b}}$ & $0.87^{\mathrm{ab}}$ & $0.78^{\mathrm{ab}}$ & $0.85^{b}$ & $0.82^{\mathrm{ab}}$ & $0.89^{a}$ \\
\hline 0.12 & & & 0.85 & $0.84^{a}$ & 0.89 & 0.80 & 0.85 & 0.83 & $0.85^{b}$ \\
\hline 0.25 & & & 0.85 & $0.81^{b}$ & 0.87 & 0.78 & 0.85 & 0.82 & $0.89^{a}$ \\
\hline \multicolumn{10}{|c|}{ P-values } \\
\hline \multicolumn{3}{|c|}{ Phytase } & $0.001^{* * *}$ & $0.007^{* *}$ & $0.003 * * *$ & $0.012^{*}$ & $0.004 * * *$ & $0.001 * * *$ & $0.059 *$ \\
\hline \multicolumn{3}{|c|}{ Lysine } & $0.069 *$ & $0.011^{*}$ & $0.025^{*}$ & 0.019* & $0.020^{*}$ & $0.003^{* * *}$ & $0.063^{*}$ \\
\hline \multicolumn{3}{|c|}{ Lysine*Phytase } & $0.003^{* *}$ & $0.0003 * * *$ & $0.005^{* * *}$ & $0.001 * * *$ & $0.049 *$ & $0.001 * * *$ & $0.005^{* *}$ \\
\hline \multicolumn{3}{|c|}{ AvP } & 0.961 & $0.028^{*}$ & 0.128 & 0.406 & 0.633 & 0.332 & $0.008 * *$ \\
\hline
\end{tabular}

and Glu with 250 and $300 \mathrm{FTU} / \mathrm{kg}$ phytase in a corn and soybean meal based diet, Agbede JH, et al. [10] did not find any positive effect adding $1500 \mathrm{FTU} / \mathrm{kg}$ phytase to sunflower and rapeseed meal testing diets with two levels of non-phytic P $(8.0$ and $4.0 \mathrm{~g} / \mathrm{kg})$. In the present study, the sampling region, the sampling method, and the marker used can partly explain the significance on amino acid digestibility. Thus, Rezvani M, et al. [15] recommended taking samples from middle and terminal sections of the ileum (between Meckel's diverticulum and ileo-caecal-colonic junction), due to almost all amino acids have been absorbed by this stage and microbial activity is low. Adedokun SA, et al. [16] advised flushing out the digesta with deionized water rather than squeezing, to reduce contamination with endogenous amino acids, meanwhile the use of titanium dioxide lead to less variability.

Ravindran V, et al. [17] reported linear increases in amino acid digestibility with increasing phytase dose when a dLys deficient diet
(1.00\%) was tested in chicks. The current experiment intended to demonstrate an increase in Lys supply by phytase, however Lys release from enzyme was not enough to compensate the reduction in dietary dLys from $0.67 \%$ to $0.77 \%$, or from 0.77 to $0.87 \%$.

Selle PH, et al. [18] reported that addition of $500 \mathrm{FTU} / \mathrm{kg}$ phytase improved performance in 7-28 day-old broilers fed a dLys deficient $(10.0 \mathrm{~g} / \mathrm{kg})$ diet, but not in a dLys adequate $(11.8 \mathrm{~g} / \mathrm{kg})$ diet. Consequently, an interaction between phytase and Lys contents was observed on the digestibility of Arg, Lys, Phe, Asp, Glu, Gly and Ser. These authors explained the mainly Lys effect based on the stimulus of epithelia transporters, such as the $\mathrm{b}^{0},+$ system at intestinal level for amino acids. Nevertheless, since Lys is the reference amino acid for ideal amino acid profile, its availability can strongly influence others essential amino acids for protein, and for that in the present Lys significatively affect amino acids avalilability. 
Table 4: Effect of addition of four doses of phytase in three graded levels of digestible lysine on apparent ileal retention of minerals in 67 wk-old White Bovans laying hens.

\begin{tabular}{|c|c|c|c|c|c|c|c|c|c|c|c|}
\hline AvP (\%) & Phytase (FTU/kg) & $\begin{array}{c}\text { Diges. } \\
\text { Lysine (\%) }\end{array}$ & $\mathbf{P}$ & $\mathrm{Ca}$ & $\mathrm{Zn}$ & $\mathrm{Fe}$ & Mg & Mn & $\mathrm{Cu}$ & $\mathbf{K}$ & $\mathrm{Na}$ \\
\hline \multirow{13}{*}{0.12} & 0 & \multirow{4}{*}{0.67} & $0.44^{b}$ & 0.37 & 0.25 & 0.15 & $0.43^{\mathrm{abc}}$ & 0.06 & 0.49 & $0.79^{\mathrm{ab}}$ & $-0.25^{c d}$ \\
\hline & 300 & & $0.44^{b}$ & 0.52 & 0.35 & 0.05 & $0.36^{\mathrm{bcd}}$ & -0.03 & 0.29 & $0.72^{\mathrm{ab}}$ & $-0.53^{d}$ \\
\hline & 1200 & & $0.57^{\mathrm{ab}}$ & 0.35 & 0.09 & 0.34 & $0.42^{\mathrm{abc}}$ & -0.18 & 0.09 & $0.78^{\mathrm{ab}}$ & $-0.02^{a b c}$ \\
\hline & 4800 & & $0.73^{\mathrm{a}}$ & 0.51 & 0.22 & 0.16 & $0.45^{\mathrm{ab}}$ & 0.04 & 0.55 & $0.86^{a}$ & $0.10^{\mathrm{abc}}$ \\
\hline & 0 & \multirow{4}{*}{0.77} & $0.45^{b}$ & 0.40 & 0.24 & 0.21 & $0.41^{\mathrm{abcd}}$ & 0.03 & 0.50 & $0.78^{a b}$ & $-0.19^{\text {cd }}$ \\
\hline & 300 & & $0.60^{\mathrm{ab}}$ & 0.66 & 0.36 & 0.37 & $0.48^{\mathrm{a}}$ & 0.20 & 0.40 & $0.82^{\mathrm{ab}}$ & $-0.23^{a b c}$ \\
\hline & 1200 & & $0.71^{\mathrm{a}}$ & 0.65 & 0.39 & 0.25 & $0.45^{a b}$ & 0.10 & 0.32 & $0.84^{\mathrm{ab}}$ & $0.27^{\mathrm{ab}}$ \\
\hline & 4800 & & $0.75^{\mathrm{a}}$ & 0.68 & 0.41 & 0.21 & $0.46^{\mathrm{ab}}$ & 0.05 & 0.44 & $0.84^{\mathrm{ab}}$ & $0.05^{\mathrm{abc}}$ \\
\hline & & & & & & & & & & & 5 \\
\hline & 0 & \multirow{5}{*}{0.87} & $0.59^{\mathrm{ab}}$ & 0.47 & 0.41 & 0.26 & $0.51^{a}$ & 0.16 & 0.42 & $0.81^{\mathrm{ab}}$ & $0.15^{a b c}$ \\
\hline & 300 & & $0.72^{\mathrm{a}}$ & 0.31 & 0.20 & 0.05 & $0.47^{\mathrm{ab}}$ & -0.03 & 0.19 & $0.82^{\mathrm{ab}}$ & $0.05^{c d}$ \\
\hline & 1200 & & $0.71^{\mathrm{a}}$ & 0.62 & 0.44 & 0.31 & $0.41^{\mathrm{abcd}}$ & 0.16 & 0.38 & $0.82^{\mathrm{ab}}$ & $0.11^{\mathrm{abc}}$ \\
\hline & 4800 & & $0.73^{\mathrm{a}}$ & 0.61 & 0.39 & 0.27 & $0.32^{\text {cd }}$ & 0.23 & 0.29 & $0.75^{a b}$ & $-0.09^{b c}$ \\
\hline \multirow[t]{2}{*}{0.25} & 0 & & $0.56^{\mathrm{ab}}$ & 0.73 & 0.50 & 0.48 & $0.31^{d}$ & 0.19 & 0.53 & $0.72^{\mathrm{b}}$ & $0.39^{\mathrm{a}}$ \\
\hline & SEM & & 0.027 & 0.063 & 0.052 & 0.051 & 0.024 & 0.066 & 0.036 & 0.015 & 0.049 \\
\hline \multicolumn{12}{|c|}{ Main Effect Means } \\
\hline \multirow{4}{*}{0.12} & 0 & & $0.50^{c}$ & 0.42 & 0.30 & 0.21 & 0.45 & 0.09 & $0.47^{\mathrm{a}}$ & 0.79 & $-0.02^{\text {cd }}$ \\
\hline & 300 & & $0.59^{\mathrm{bc}}$ & 0.50 & 0.30 & 0.16 & 0.44 & 0.05 & $0.19^{b}$ & 0.79 & $-0.24^{d}$ \\
\hline & 1200 & & $0.66^{\mathrm{ab}}$ & 0.54 & 0.30 & 0.30 & 0.43 & 0.03 & $0.37^{a}$ & 0.81 & $0.12^{\mathrm{b}}$ \\
\hline & 4800 & & $0.74^{\mathrm{a}}$ & 0.60 & 0.34 & 0.22 & 0.41 & 0.11 & $0.43^{\mathrm{a}}$ & 0.81 & $0.02^{\mathrm{bc}}$ \\
\hline 0.25 & 0 & & $0.56^{\mathrm{bc}}$ & 0.73 & 0.50 & 0.48 & 0.31 & 0.19 & $0.53^{\mathrm{a}}$ & 0.72 & $0.39^{\mathrm{a}}$ \\
\hline \multirow{3}{*}{0.12} & & 0.67 & $0.55^{b} \mathbf{L}$ & 0.44 & $0.23^{b}$ & 0.18 & 0.42 & -0.03 & 0.36 & 0.78 & $-0.17^{c} \mathbf{L}$ \\
\hline & & 0.77 & $0.63^{\mathrm{ab}} \mathbf{Q}$ & 0.60 & $0.35^{\mathrm{ab}}$ & 0.26 & 0.45 & 0.10 & 0.41 & 0.82 & $0.040^{\mathrm{b}} \mathbf{Q}$ \\
\hline & & \multirow{2}{*}{0.87} & $0.69^{\mathrm{a}} \mathbf{Q}$ & 0.50 & $0.36^{\mathrm{a}}$ & 0.22 & 0.43 & 0.13 & 0.32 & 0.80 & $-0.02^{b}$ \\
\hline 0.25 & & & $0.56^{\mathrm{ab}}$ & 0.73 & $0.50^{\mathrm{a}}$ & 0.48 & 0.31 & 0.19 & 0.53 & 0.72 & $0.39^{\mathrm{a}}$ \\
\hline 0.12 & & & 0.62 & $0.51^{\mathrm{b}}$ & $0.31^{\mathrm{b}}$ & $0.22^{\mathrm{b}}$ & $0.43^{\mathrm{a}}$ & 0.07 & $0.36^{b}$ & $0.80^{\mathrm{a}}$ & $-0.05^{b}$ \\
\hline 0.25 & & & 0.56 & $0.73^{\mathrm{a}}$ & $0.50^{\mathrm{a}}$ & $0.48^{\mathrm{a}}$ & $0.31^{b}$ & 0.19 & $0.53^{\mathrm{a}}$ & $0.72^{\mathrm{b}}$ & $0.39^{a}$ \\
\hline \multicolumn{12}{|c|}{$p$-values } \\
\hline \multicolumn{3}{|c|}{ Phytase } & $<.0001$ & 0.204 & 0.951 & 0.277 & $0.579 \mathbf{Q}$ & 0.844 & $<.0001$ & $0.483 \mathrm{~L}$ & $<.0001$ \\
\hline \multicolumn{3}{|c|}{ Lysine } & $0.001^{* * *}$ & 0.131 & 0.079 & 0.401 & 0.503 & 0.129 & 0.145 & 0.155 & $0.002 * *$ \\
\hline \multicolumn{3}{|c|}{ Lysine*Phytase } & $0.062^{*}$ & 0.393 & 0.131 & 0.201 & $0.035^{*}$ & 0.452 & 0.093 & $0.008 * *$ & $0.001^{* * *}$ \\
\hline \multicolumn{3}{|c|}{ AvP } & 0.226 & $0.059 *$ & $0.048^{*}$ & $0.006 * *$ & $0.006 * *$ & 0.298 & $0.011^{*}$ & $0.003 * *$ & $<.0001$ \\
\hline
\end{tabular}

Hydrolyses of Phyt-ac was reported to increase by $50 \%$ and $66 \%$, respectively, adding 250 and $500 \mathrm{FTU} / \mathrm{kg}$ of a 3-phytase to laying hen diets [19], meanwhile in another study, ileal P digestibility showed improvements of $39.0 \%, 44.6 \%$, and $51.3 \%$ with 150,300 , and 450 FTU/ $\mathrm{kg} 3$-phytase, respectively [20]. In our study, increasing levels of phytase improved the release of $\mathrm{P}$ from diets, but higher doses were required to release higher levels of phytate-P. On the other hand, Ca digestibility was not influenced by phytase, notwithstanding of modifications in $\mathrm{P}$ digestibility influence $\mathrm{Ca}$ absorption, for maintaining the optimal $\mathrm{Ca}: \mathrm{P}$ ratio in biological process such as egg shell formation.
No consistent effects of phytase were observed in this study on $\mathrm{Zn}$, $\mathrm{Cu}, \mathrm{Co}, \mathrm{Mn}, \mathrm{Mg}$, and Fe digestibility, in spite of chelation process of these minerals by Phyt-ac [21], likely because contents in diets was covering the mineral requirements of the hens, disguising phytase improvements on extra availability. In the case of $\mathrm{Na}$, intestinal mucus production increases in the presence of phytate, which can result in higher losses of mucus component, including $\mathrm{Na}$ [22]. When an exogenous phytase is added, a direct effect on $\mathrm{Na}$ homeostasis is expected, such as was observed in the present study. Cowieson A, et al. [4] reported that including $1000 \mathrm{FTU} / \mathrm{kg}$ phytase offset the 
Table 5: Effect of addition of four doses of phytase in three graded levels of digestible lysine on content of hydrolyses molecules of phytic acid in gizzard digesta and coenzyme Q10a in liver of 65 week-old White Bovans laying hens.

\begin{tabular}{|c|c|c|c|c|c|c|c|c|c|}
\hline \multirow{2}{*}{$\mathbf{t}$} & \multirow{2}{*}{ Phytase (FTU/kg) } & \multirow{2}{*}{ Diges. Lysine (\%) } & Ins6 $P^{c}$ & Ins5P & Ins4P & Ins3P & Ins2P & Inositol & \multirow[t]{2}{*}{ CoQ10 } \\
\hline & & & \multicolumn{6}{|c|}{ N mol/g Dry Weight } & \\
\hline \multirow{12}{*}{0.12} & 0 & \multirow{4}{*}{0.67} & 4159 & 400 & $3^{d}$ & 557 & $167^{d}$ & 656 & $392.9^{b}$ \\
\hline & 300 & & 2108 & 259 & $93^{\text {cd }}$ & 554 & $546^{b}$ & 871 & $338.8^{\mathrm{bc}}$ \\
\hline & 1200 & & 1859 & 205 & $45^{\text {cd }}$ & 383 & $394^{\mathrm{bcd}}$ & 103 & $380.2^{b}$ \\
\hline & 4800 & & 489 & 43 & $15^{d}$ & 328 & $207^{c d}$ & 629 & --- \\
\hline & 0 & \multirow{4}{*}{0.77} & 2991 & 362 & $49^{\text {cd }}$ & 411 & $321^{b c d}$ & 293 & --- \\
\hline & 300 & & 1627 & 192 & $83^{\text {cd }}$ & 380 & $373^{\mathrm{bcd}}$ & 450 & --- \\
\hline & 1200 & & 701 & 78 & $34^{\text {cd }}$ & 318 & $493^{\mathrm{bc}}$ & 78 & --- \\
\hline & 4800 & & 326 & 43 & $5^{d}$ & 303 & $452^{\text {bcd }}$ & 841 & --- \\
\hline & 0 & \multirow{5}{*}{0.87} & 3260 & 640 & $769^{a}$ & 648 & $921^{\mathrm{a}}$ & 441 & $607.5^{a}$ \\
\hline & 300 & & 2424 & 428 & $334^{b}$ & 511 & $911^{a}$ & 572 & $\mathrm{c} 300.5^{\mathrm{bcd}}$ \\
\hline & 1200 & & 1511 & 252 & $260^{\mathrm{bc}}$ & 376 & $963^{a}$ & 1078 & $217.5^{d}$ \\
\hline & 4800 & & 1480 & 283 & $123^{\mathrm{bcd}}$ & 350 & $1034^{a}$ & 1145 & --- \\
\hline 0.25 & 0 & & 4187 & 827 & $192^{b c d}$ & 364 & $882^{a}$ & 119 & $257.0^{\text {cd }}$ \\
\hline \multicolumn{3}{|r|}{ SEM } & 253.5 & 44.07 & 26.80 & 26.70 & 37.27 & 103.5 & 25.51 \\
\hline \multicolumn{10}{|c|}{ Main Effect Means } \\
\hline \multirow{4}{*}{0.12} & 0 & & $3470^{\mathrm{a}}$ & $467^{b}$ & $273^{a}$ & $539^{a}$ & $470^{\mathrm{b}}$ & $463^{b c}$ & $500.2^{\mathrm{a}}$ \\
\hline & 300 & & $2053^{b}$ & $293^{b c}$ & $170^{\mathrm{ab}}$ & $482^{\mathrm{ab}}$ & $610^{\mathrm{b}}$ & $631^{\mathrm{abc}}$ & $319.7^{b}$ \\
\hline & 1200 & & $1357^{\mathrm{bc}}$ & $178^{c}$ & $113^{b c}$ & $359^{c}$ & $617^{\mathrm{b}}$ & $963^{a}$ & $298.8^{b}$ \\
\hline & 4800 & & $765^{c}$ & $123^{c}$ & $48^{c}$ & $327^{c}$ & $564^{b}$ & $871^{\mathrm{ab}}$ & --- \\
\hline 0.25 & 0 & & $4187^{a}$ & $827^{a}$ & $192^{\mathrm{abc}}$ & $364^{\mathrm{bc}}$ & $882^{a}$ & $119^{c}$ & $257.0^{b}$ \\
\hline \multirow{3}{*}{0.12} & & 0.67 & $2154^{b}$ & $227^{c}$ & $39^{c}$ & $456^{a}$ & $329^{b}$ & 797 & 370.6 \\
\hline & & 0.77 & $1411^{b}$ & $169^{c}$ & $43^{c}$ & $353^{b}$ & $410^{\mathrm{b}}$ & 591 & --- \\
\hline & & \multirow{2}{*}{0.87} & $2169^{b}$ & $401^{b}$ & $372^{a}$ & $471^{\mathrm{a}}$ & $957^{\mathrm{a}}$ & 809 & 375.1 \\
\hline 0.25 & & & $4187^{a}$ & $827^{a}$ & $192^{b}$ & $364^{\mathrm{ab}}$ & $882^{a}$ & 119 & 257.0 \\
\hline 0.12 & & & $1911^{b}$ & $265^{b}$ & 151 & 427 & $565^{b}$ & $732^{a}$ & $372.9^{a}$ \\
\hline 0.25 & & & $4187^{a}$ & $827^{a}$ & 192 & 364 & $882^{\mathrm{a}}$ & $119^{b}$ & $257.0^{\mathrm{b}}$ \\
\hline \multicolumn{10}{|c|}{ p-values } \\
\hline \multicolumn{3}{|c|}{ Phytase } & $<.0001 \mathrm{Q}$ & $<.0001 \mathrm{Q}$ & $<.0001 \mathrm{~L}$ & $<.0001 \mathrm{Q}$ & $0.025^{*}$ & $0.005^{* *} \mathbf{Q}$ & $<.0001 \mathrm{~L}$ \\
\hline \multicolumn{3}{|c|}{ Lysine } & $0.025^{*}$ & 0.001 & $<.0001 \mathrm{~L}$ & 0.001 & $<.0001 \mathrm{~L}$ & 0.156 & 0.831 \\
\hline \multicolumn{3}{|c|}{ Lysine*Phytase } & 0.469 & 0.879 & $<.0001$ & 0.289 & $0.011^{*}$ & 0.314 & $<.0001$ \\
\hline \multicolumn{3}{|c|}{ AvP } & $<.0001$ & $<.0001$ & 0.392 & 0.191 & $<.0001$ & $0.002 * *$ & 0.001 \\
\hline
\end{tabular}

${ }^{a}$ Content of coenzyme Q10 is reported in three phytase doses and two levels of digestible lysine

${ }^{b}$ Coenzyme Q10

'Low inositol ester

Statistically significant $* p \leq 0.05, * * p \leq 0.01, * * * p \leq 0.001$. a-b Show significant differences among treatment means.

negative impact of Phyt-ac on Na excretion in broilers; whereas, they did not observe any changes in the excretion of $\mathrm{Zn}, \mathrm{Fe}, \mathrm{Mg}, \mathrm{Mn}$, and $\mathrm{Cu}$. Moreover, the likely influence on $\mathrm{Na} / \mathrm{K}$ ATPase intestinal pump by changes in $\mathrm{Na}$ and $\mathrm{K}$ digestibility, could affect general nutrient absorption, other than $\mathrm{P}$, as was obtained.

In poultry, Phyt-ac degradation increases with increasing phytase dose, with a linear positive relation observed in broilers and also in laying hens [9]. Persson $\mathrm{H}$, et al. [21] reported a lower binding strength for $\mathrm{Cu}, \mathrm{Zn}$ and $\mathrm{Cd}$ when Ins-4 and $3 \mathrm{P}$ were tested compare to Ins-5 and 6P. Additionally, $\mathrm{Xu}$ P, et al. [23] observed in vitro that $\mathrm{Ca}$ and $\mathrm{Zn}$ solubilities were increased when Phyt-ac was degraded to Ins-3P. In the current study, $300 \mathrm{FTU} / \mathrm{kg}$, which is generally the standard dose of phytase, was efficient in the initial breakdown of Phyt-ac, meanwhile $1200 \mathrm{FTU} / \mathrm{kg}$ increased free inositol release. Thus, high phytase doses 
increased the presence of Ins-P breakdown products with weaker covalent binding strength, and then the availability of nutrients that are susceptible to chelation by Phyt-ac.

One important result to highlight in this study was the effect of dLys on Phyt-ac degradation by phytase; this can be explained following the electrostatic interactions between molecules. Kaup SM and Greger J [24] investigated the impact chloride salt type on intestine absorption, bone deposition, and excretion of $\mathrm{P}, \mathrm{Ca}$ and $\mathrm{Mg}$ in rats, concluding that Lys chloride was able to increase Ca excretion. Also, when Lys chloride and $\mathrm{Ca}$ were supplied in the diet, $\mathrm{P}$ and $\mathrm{Mg}$ absorption in the intestine and $\mathrm{Mg}$ deposition in bone were affected negatively. Thus, anions such as chloride can influence the electrostatic interactions among dietary molecules, depending of type of Ins-P present, and can result in deeply changes in digestive process.

Therefore, in the present study Lys chloride addition into the diet could have altered the solubility and hydrolysis of Phyt-ac, resulting in mainly effects of dLys level into the diets. Similarly, Banks K, et al. reported linear reductions in $\mathrm{P}$ retention with increasing dietary $\mathrm{Cu}$ concentration from 125 to $375 \mathrm{ppm}$, but not for 10 and $62.5 \mathrm{ppm}$ [25]. Furthermore, Wilcock P and Walk CL [26] reported that Cu form included in diet influences the solubility of $\mathrm{Cu}$-phytate complexes, since sulfate, chloride and tribasic chloride $\mathrm{Cu}$ resulted in higher levels of insoluble $\mathrm{Cu}$ than citrate and lysine sources at $\mathrm{pH}$ 5.5. This reinforces the idea that molecular composition highly influences Phytac stability and susceptibility to phytase action.

Laying hens produce phosphatases able to hydrolyze Phyt-ac up to $35 \%$ to releasing inositol and free phosphate groups [27]. Truong $\mathrm{HH}$, et al. [28] reported $63 \%$ overall hydrolysis of phytate in broilers, with $24 \%$ corresponded to endogenous enzymes action and 39\% to $1000 \mathrm{FTU} / \mathrm{kg}$ for an exogenous phytase. Recently, Wilcock P and Walk CL [26] established that using $1500 \mathrm{FTU} / \mathrm{kg}$ phytase, a high dose in poultry meat production, more than $85 \%$ of Phyt-ac is hydrolyzed, meanwhile the standard dose of $500 \mathrm{FTU} / \mathrm{kg}$ degrades $29 \%$ or $34 \%$. In our study, inclusion of 4800,1200 and $300 \mathrm{FTU} / \mathrm{kg}$ phytase reduced Ins-P content by $78 \%, 61 \%$, and $41 \%$, respectively, in gizzard digesta.

Supplementation with $0.13 \%$ inorganic $\mathrm{P}$ was not equivalent in $\mathrm{P}$ release by the phytase doses evaluated in the current trial. Liu N, et al. [29] found higher P digestibility using $0.28 \%$ avP rather than including $300 \mathrm{FTU} / \mathrm{kg}$ from three different commercial phytase in Hy-line Brown layers fed $0.58 \%$ dLys. By contrast, in the present study $300 \mathrm{FTU} / \mathrm{kg}$ phytase gave a higher P digestibility (59\%) than inorganic-P inclusion (56\%), and while $\mathrm{P}$ was more available with increasing phytase dose, the effect was lower in diets with $0.87 \%$ dLys. Furthermore, the addition of $0.13 \% \mathrm{P}$ as monocalcium phosphate increased the presence of Ins-6 and 5P in gizzard digesta and reduced Ins-2P and inositol concentrations. Shastak Y, et al. [30] found less Ins-6P hydrolysis in ileum digesta and excreta of 20-24 day-old broilers with increasing inorganic-P from $0.075 \%$ to $0.15 \%$ using monocalcium phosphate monohydrate. Similarly, Li W, et al. [31] observed an increase of Ins-6P presence in proventriculus and gizzard digesta in 13 day old chickens with $0.45 \%$ non-phytate $\mathrm{P}$ relative to $0.28 \%$ non-phytate $\mathrm{P}$ when fed a mixed basal diet with monocalcium phosphate as inorganic $\mathrm{P}$ source. Inorganic $\mathrm{P}$ content is one of the most important negative factors in phytase hydrolysis; hence, the low inorganic-P level in the current trial $(0.12 \%)$ allowed phytase to have a significant effect on amino acid digestibility, as previously reported with 0,250 and $500 \mathrm{FTU} / \mathrm{kg}$ for amino acid and $P$ digestibility [19].

High concentrations of cell CoQ10 content is indicative of oxidative status, mainly due to catabolism of lipids, carbohydrates and proteins
[8].In the diet with $0.87 \%$ dLys without phytase, more catabolism of extra amino acid was presented altering the citric acid cycle where CoQ10 is a key molecule. Conversely, CoQ10 content showed less variation to phytase in $0.67 \%$ dLys because the enzyme can regulate the requirements of $\mathrm{P}$ but not the lysine. In the $0.87 \%$ dLys diet, inorganic $\mathrm{P}$ supplementation and phytase addition decreased liver CoQ10 concentration by $58 \%$, although the greatest effect was observed with $1200 \mathrm{FTU} / \mathrm{kg}$ addition.

Karadas F, et al. (2010) observed a higher liver content of CoQ10 and better performance in broilers fed diets with $500 \mathrm{FTU} / \mathrm{kg}(99.95$ $\mu \mathrm{g} / \mathrm{g})$ and $12500 \mathrm{FTU} / \mathrm{kg}(119.7 \mathrm{ug} / \mathrm{g})$ of phytase compared to 250 FTU/kg (63.86 ug/g) [7]. Additionally, Karadas F, et al. [32] reported no change in the coenzyme concentration using $12500 \mathrm{FTU} / \mathrm{kg}$ or $\mathrm{P}$ inorganic supplementation (129.9 and $119.7 \mathrm{ug} / \mathrm{g}$, respectively). The above-mentioned author assumed that high CoQ10 enhances oxidative status of cellular membranes in the liver, and it allows better productive performance. In our case, opposite result was obtained, and it is likely due to differences in intrinsic physiologically characteristics of layer as age, but no previous report are available to confirm the current results. The only available research quantifying CoQ10 content reported improvement of $8 \%$ when a source of CoQ10 was supplemented at diet [33].

In conclusion, the increase of phytase dose has improved the availability of essential, non-essential amino acids, $\mathrm{P}$ and $\mathrm{Na}$, with less effect on high dLys diet. Moreover, higher inclusion promotes the presence of lower inositol esters (Ins5-2P) and inositol from Phytac, and affect CoQ10 content in liver. The response to inorganic P addition contrasted to that of standard dose of phytase (300 FTU/kg), even though for $\mathrm{P}$ digestibility.

\section{Acknowledgments}

The authors express their gratitude to the DGAPA and PAPIIT (project IN 214015) for financial support of this project.

\section{References}

1. Cheryan M (1980) Phytic acid interactions in food systems. Crit Rev Food Sci Nutr 13: 297-335.

2. Vohra P, Gray GA, Kratzer FH (1965) Phytic acid-metal complexes. Proceedings of the Society for Experimental Biology and Medicine 120: 447-449.

3. Konietzny U, Greiner R (2002) Molecular and catalytic properties of phytate degrading enzymes (phytases). Int J Food Sci Tech 37: 791812 .

4. Cowieson A, Acamovic T, Bedford M (2004) The effects of phytase and phytic acid on the loss of endogenous amino acids and minerals from broiler chickens. Br Poult Sci 45: 101-108.

5. Walk CL, Santos TT, Bedford MR (2014) Influence of Superdoses of a Novel Microbial Phytase on Growth Performance, Tibia Ash, and Gizzard Phytate and Inositol in Young Broilers. Poult Sci 93: 11721177.

6. Cowieson AJ, Wilcock P, Bedford MR (2011) Super-dosing effects of phytase in poultry and other monogastrics. World Poultry Sci J 67: 225-236.

7. Karadas F, Pirgozliev V, Pappas AC, Acamovic T, Bedford MR (2010) Effects of Different Dietary Phytase Activities on the Concentration of Antioxidants in the Liver of Growing Broilers. J Anim Physiol Anim Nutr 94: 519-526. 
8. Pisoschi AM, Pop A (2015) The role of antioxidants in the chemistry of oxidative stress: A review. Eur J Med Chem 97: 55-74.

9. Hughes A, Dahiya J, Wyatt C, Classen H (2009) Effect of Quantum Phytase on Nutrient Digestibility and Bone Ash in White Leghorn Laying Hens Fed Corn-Soybean Meal-Based Diets. Poult Sci 88: 1191-1198.

10. Agbede JH, Kluth C, Rodehutscord M (2009) Amino acid digestibility and energy metabolisability as affected by dietary calcium and supplemented phytase in caecectomised laying hens. Arch Geflügelk 73: 73-79.

11. Silva EP, Malheiros EB, Sakomura NK, Venturini KS, Hauschild L, et al. (2015) Lysine requirements of laying hens. Livest Sci 173: 69-77.

12. Blaabjerg K, Hansen-Møller J, Poulsen HD (2010) High-performance ion chromatography method for separation and quantification of inositol phosphates in diets and digesta. J Chromatogr B Analyt Technol Biomed Life Sci 878: 347-354.

13. Mattila $P$, Kumpulainen J (2001) Coenzymes $Q_{9}$ and $Q_{10}$ : Contents in Foods and Dietary Intake. J Food Compos Anal 14: 409-417.

14. Jalal MA, Scheideler SE, Wyatt C (1999) Effect of phytase supplementation on egg production parameters and amino acid digestibility. Poult Sci 45: 123-125.

15. Rezvani M, Kluth H, Elwert C, Rodehutscord M (2008) Effect of lleum Segment and Protein Sources on Net Disappearance of Crude Protein and Amino Acids in Laying Hens. Br Poult Sci 49: 28-36.

16. Adedokun SA, Adeola O, Parsons CM, Lilburn MS, Applegate TJ (2011) Factors Affecting Endogenous Amino Acid Flow in Chickens and the Need for Consistency in Methodology. Poult Sci 90: 1737 1748.

17. Ravindran V, Selle P, Ravindran G, Morel P, Kies A, et al. (2001) Microbial Phytase Improves Performance, Apparent Metabolizable Energy, and Ileal Amino Acid Digestibility of Broilers Fed a LysineDeficient Diet. Poult Sci 80: 338-344.

18. Selle PH, Ravindran V, Ravindran G, Bryden WL (2007) Effects of dietary lysine and microbial phytase on growth performance and nutrient utilisation of broiler chickens. Asian-Aust. J Anim Sci 20 1100-1107.

19. Van der Klis J, Versteegh H, Simons P, Kies A (1997) The efficacy of phytase in corn-soybean meal-based diets for laying hens. Poult Sci76: 1535-1542.

20. Francesch M, Broz J, Brufau J (2005) Effects of an Experimental Phytase on Performance, Egg Quality, Tibia Ash Content and Phosphorus Bioavailability in Laying Hens Fed on Maize- Or BarleyBased Diets. Br Poult Sci 46: 340-348.

21. Persson $\mathrm{H}$, Türk $\mathrm{M}$, Nyman $\mathrm{M}$, Sandberg AS (1998) Binding of $\mathrm{Cu}^{2+}$, $\mathrm{Zn}^{2+}$, and $\mathrm{Cd}^{2+}$ to Inositol Tri-, Tetra-, Penta-, and Hexaphosphates. $J$ Agric Food Chem 46: 3194-3200.
22. Selle PH, Cowieson AJ, Cowieson NP, Ravindran V (2012) Proteinphytate interactions in pig and poultry nutrition: a reappraisal. Nutr Res Rev 25: 1-17.

23. Xu P, Price J, Wise A, Aggett PJ (1992) Interaction of inositol phosphates with calcium, zinc, and histidine. J Inorg Biochem 47: 119-130.

24. Kaup SM, Greger J (1990) Effect of Various Chloride Salts on the Utilization of Phosphorus, Calcium, and Magnesium. J Nutr Biochem 1: $542-548$

25. Banks K, Thompson K, Jaynes P, Applegate T (2004) The Effects of Copper on the Efficacy of Phytase, Growth, and Phosphorus Retention in Broiler Chicks. Poult Sci 83: 1335-1341.

26. Wilcock P, Walk CL (2016) Low phytate nutrition-what is the pig and poultry industry doing to counter dietary phytate as an anti-nutrient and how is it being applied? In: Walk CL, Kuhn I, Stein HH, Kidd MT, Rodehutscord M (eds) Phytate Destruction Consequences for Precision Animal Nutrition. Wageningen Academic Publishers, The Netherlands 87-106.

27. Maenz DD, Classen HL (1998) Phytase activity in the small intestinal brush border membrane of the chicken. Poult Sci 77: 557-563.

28. Truong HH, Yu S, Peron A, Cadogan DJ, Khoddami A, et al. (2014) Phytase supplementation of maize-, sorghum-and wheat-based broiler diets with identified starch pasting properties influences phytate $\left(\mathrm{IP}_{6}\right)$ and sodium jejunal and ileal digestibility. Animal Feed Scienceand Techology 198: 248-256.

29. Liu N, Liu G, Li F, Sands J, Zhang S, et al. (2007) Efficacy of Phytases on Egg Production and Nutrient Digestibility in Layers Fed Reduced Phosphorus Diets. Poult Sci 86: 2337-2342.

30. Shastak $Y$, Zeller E, Witzig M, Schollenberger M, Rodehutscord M (2014) Effects of the Composition of the Basal Diet on the Evaluation of Mineral Phosphorus Sources and Interactions with Phytate Hydrolysis in Broilers. Poult Sci 93: 2548-2559.

31. Li W, Angel R, Kim SW, Brady K, Yu S, et al. (2016) Impacts of dietary calcium, phytate, and nonphytate phosphorus concentrations in the presence or absence of phytase on inositol hexakisphosphate $\left(\mathrm{IP}_{6}\right)$ degradation in different segments of broilers digestive tract. Poult Sci 95: 581-589.

32. Karadas F, Pirgozliev V, Acamovic T, Bedford MR (2005) The effects of dietary phytase activity on the concentration of Coenzyme $Q_{10}$ in the liver of young turkeys and broilers. British Poultry Abstract 1: $1-2$.

33. Kamisoyama H, Honda K, Kitaguchi K, Hasegawa S (2010) Transfer of dietary coenzyme $\mathrm{Q}_{10}$ into the egg yolk of laying hens. J Poult Sci 47: 28-33. 